\title{
Diabetes and risk of subsequent gastric cancer: a population-based cohort study in Taiwan
}

\author{
Yu-Lung Chen $\cdot$ Kao-Chi Cheng $\cdot$ Shih-Wei Lai $\cdot$ \\ I-Ju Tsai $\cdot$ Che-Chen Lin $\cdot$ Fung-Chang Sung • \\ Cheng-Chieh Lin $\cdot$ Pei-Chun Chen
}

Received: 8 March 2012/ Accepted: 5 September 2012/Published online: 5 October 2012

(c) The International Gastric Cancer Association and The Japanese Gastric Cancer Association 2012

\begin{abstract}
Background Epidemiological data concerning the association between diabetes and the subsequent development of gastric cancer are controversial. This population-based retrospective cohort study investigated the subsequent risk of gastric cancer for diabetic patients.

Methods From claims data of the universal health insurance of Taiwan, we identified 19,625 persons aged $\geq 20$ years newly diagnosed with diabetes during 2000-2005. A comparison group $(n=78,500)$, frequency matched by age, sex, and calendar year, was randomly selected from
\end{abstract}

Y.-L. Chen and K.-C. Cheng contributed equally to this article.

Y.-L. Chen · K.-C. Cheng · S.-W. Lai - Cheng-Chieh Lin

Department of Family Medicine, China Medical University

Hospital, Taichung 404, Taiwan

e-mail: yulung64@pchome.com.tw

Y.-L. Chen

Taichung Prison Peide Hospital, Taichung 408, Taiwan

S.-W. Lai · Cheng-Chieh Lin

School of Medicine, China Medical University, Taichung 404,

Taiwan

I.-J. Tsai · Che-Chen Lin - P.-C. Chen

Management Office for Health Data, China Medical University

Hospital, Taichung 404, Taiwan

F.-C. Sung

Department of Public Health, China Medical University,

Taichung 404, Taiwan

P.-C. Chen $(\bowtie)$

Graduate Institute of Clinical Medical Science, College of Public

Health, China Medical University, 91 Hsueh-Shih Road,

Taichung 404, Taiwan

e-mail: peichun@mail.cmu.edu.tw people without diabetes. Incidence and hazard ratios (HR) of gastric cancer were ascertained during the follow-up period until 2008. We also explored associations of antidiabetic medicines with the incidence of gastric cancer.

Results During the follow-up period, 47 subjects in the diabetic group and 216 subjects in the comparison group suffered gastric cancer, with the incidence rates of 4.34 and 4.86 per 10,000 person-years, respectively. During the first 4 years after diabetes diagnosis, the incidence of gastric cancer was relatively low in diabetic patients [adjusted $\mathrm{HR}=0.63 ; 95 \%$ confidence interval $(\mathrm{CI})=0.42-0.97]$. However, after that time, the diabetic group had a $76 \%$ $(95 \% \mathrm{CI}=1.06-2.91)$ higher risk of developing gastric cancer than the comparison group. In diabetic patients, alpha-glucosidase inhibitors were associated with a significantly decreased risk of gastric cancer (adjusted HR $=0.38$; $95 \% \mathrm{CI}=0.15-0.96$ ).

Conclusions Our findings suggested that the association between diabetes and subsequent risk of gastric cancer may vary over time. Increased risk of gastric cancer was observed in patients with longer duration of diabetes.

Keywords Diabetes mellitus - Stomach neoplasms · Cohort studies $\cdot$ Hypoglycemic agents

\section{Introduction}

Stomach cancer is the fourth most prevalent cancer in the world [1]. Although mortality from stomach cancer has decreased in many countries during the past decade, it is still the second leading cause of death from cancer worldwide [1-3]. In Taiwan, the mortality rate from gastric cancer in the general population decreased from 1995 to 
2006, although gastric cancer morbidity remains high among the population [3].

Diabetes and cancer are common diseases. The cooccurrence of the two diseases has frequently drawn attention in clinical practice and has been discussed for decades $[4,5]$. Epidemiological studies have revealed that preexisting diabetes may increase mortality in patients with cancer [6]. Diabetes has been associated with increased risk of incidence of cancer at several sites [4], including cancer of the liver [7-14], kidney [7, 10-12], endometrium [10, $11,15]$, pancreas [7-10, 12-15], and colon and rectum [8, $10,12-14]$. However, the association between diabetes and risk of gastric cancer is inconclusive [7, 8, 11, 12, 16], and the association may vary among ethnic groups [12]. Limited studies have also expressed concern whether antidiabetic pharmacological therapy affects gastric cancer risk for patients with diabetes $[10,11]$.

Using the National Health Insurance Research Database (NHIRD), a national representative claims database in Taiwan, this study was undertaken to examine whether diabetes is associated with increased risk of gastric cancer. We also explored the association of antidiabetic medications with the incidence of gastric cancer.

\section{Materials and methods}

\section{Source of data}

The NHIRD, a computerized claims database of Taiwan National Health Insurance (NHI), was obtained from the National Health Research Institute for this cohort study. Covering $99 \%$ of the residents of Taiwan, the NHI is a universal health insurance program that has provided health care to people of all ages since 1995 [17]. All beneficiaries are free to choose their healthcare providers among the contracted institutions, which represent more than $90 \%$ of healthcare institutions in Taiwan. In this study, we used a subset of NHIRD containing 1 million people randomly selected from the total 23 million beneficiaries enrolled in NHI. The datasets contain a registry of catastrophic illness patients, inpatient claims, ambulatory care claims, and prescriptions, as well as the registration files, which include demographic variables such as age and sex, date of registration, and termination of coverage. The datasets contain medical claims and information on registration from 1996 to 2008 for each of the 1 million people, and thus formed a longitudinal database allowing us to observe the association between diabetes and subsequent gastric cancer. Personal identifications were encrypted before the release of the database to safeguard patient confidentiality and privacy.
Study subjects

We identified patients with an initial diagnosis of diabetes mellitus (The International Classification of Diseases, Ninth Revision, Clinical Modification, ICD-9 code 250) being treated with antidiabetic drugs during 2000-2005 ( $N=20,382)$, excluding those younger than 20 years of age $(n=156)$, those with a history of cancer (ICD-9 codes 140-208) $(n=596)$, and those who withdrew from NHI in the year identified $(n=5)$. A patient was defined as receiving treatment with antidiabetic drugs if he or she had received at least one prescription among the following drugs: metformin, sulfonylureas, thiazolidinediones, alphaglucosidase inhibitors, nateglinide, repaglinide, and insulin. The study index date was defined as the first date of prescription with antidiabetic therapy. A total of 19,625 patients formed the diabetic group. A comparison group frequency matched on age (every 5 years), sex, and index year of diabetic group was randomly selected from people who had never been diagnosed with diabetes and cancer by using a control-to-case ratio of $4(N=78,500)$. Comorbidities related to the risk of gastric cancer were identified, including Helicobacter infection (ICD-9 code 041.86), non-acute gastritis (which consisted of all gastritis diagnoses except acute gastritis) (ICD-9 codes 535.1-535.4, 535.7), pernicious anemia (ICD-9 code 281.0), gastric polyp (ICD-9 code 211.1), vagotomy (ICD-9 codes 44.00, 44.01, 44.02, 44.03), partial gastrectomy (ICD-9 codes 43.89, 43.6, 43.5, 43.7, 43.81), gastroesophageal reflux disease (GERD) (ICD-9 codes 530.81, 530.11), gastric ulcer (ICD-9 code 531), obesity (ICD-9 codes 278.00, 278.01, 278.1), asbestosis (ICD-9 code 501), and pneumoconiosis (ICD-9 codes 500, 502, 503, 504, 505). All subjects were followed up from the index date until the date of occurrence of gastric cancer (ICD-9 code 151), of withdrawal from the NHI, or December 31, 2008, whichever occurred first. A patient was identified as having gastric cancer if he or she has registered gastric cancer as a catastrophic illness. In the NHI program, the diagnosis of major critical diseases, including gastric cancer, entitles the beneficiaries to an exemption from copayments for healthcare related to the disease. Thus, patients may register as having catastrophic illness after their physicians have confirmed the diagnosis.

\section{Statistical analysis}

We compared demographic status between the diabetic patients and the comparison cohort using the chi-square test. We plotted Kaplan-Meier curves to compare the cumulative incidence of gastric cancer between patients with diabetic and the nondiabetic comparison group during the follow-up period. Cox proportional regression analysis 
measuring hazard ratio (HR) and $95 \%$ confidence interval (CI) was used to assess the association between diabetes and the risk of developing gastric cancer. Comorbidity was included in covariates to account for potential confounding effects. We used SAS software (version 9.1 for Windows; SAS Institute, Cary, NC, USA) for all statistical analyses. A two-sided $P$ value less than 0.05 was considered statistically significant. Kaplan-Meier survival curves were plotted using R (version 2.11.1; R Development Core Team, Boston, MA, USA, 2010).

\section{Results}

Among the study subjects, men and insured persons 40-64 years of age were dominant (Table 1). Patients with diabetes tended to be likely to have non-acute gastritis, gastric ulcer, obesity, and GERD (all $P<0.0001$, except for $P=0.002$ for GERD), relative to the comparison group, but were less likely to have vagotomy $(P=0.02)$.

During a median follow-up period of 5.5 years for both groups, the incidence of gastric cancer was slightly lower in the diabetic group than in the comparison group [4.34 and 4.86 per 10,000 person-years; adjusted HR (95\% CI), $0.90(0.65-1.23)]$, representing 47 and 216 cases of gastric cancer, respectively (Table 2). Kaplan-Meier curves of event-free survival for gastric cancer showed that the event-free rate was higher in the diabetes group during earlier years but was higher in the comparison group after 7-8 years (Fig. 1), suggesting that the proportional hazard assumption may not hold. We further assessed the assumption by including an interaction term of diabetes and the log of survival time in the Cox regression model, and the results suggested violation of the assumption ( $P$ value of the product term, 0.034). Thus, we partitioned the follow-up time year by year into nine periods and repeated the Cox model to observe the changes in risk over time. The risk for incidence of gastric cancer decreased with the diabetes group compared with the nondiabetic group during the first 4 years and increased beyond 5 years after the diagnosis of diabetes (Fig. 2). HR increased to a greater degree after 6 years. In analysis stratified by follow-up duration at 5 years (Table 2), the HR (95\% CI) for the diabetes group compared with the nondiabetic group was $0.63(95 \% \mathrm{CI}=0.42-0.97)$ in the first 4 years after diabetes diagnosis and $1.76(95 \% \quad \mathrm{CI}=1.06-2.91)$ thereafter.

Table 3 shows the characteristics and comorbidities of patients in association with risk of developing gastric cancer. The risk of gastric cancer was higher in men than in

Table 1 Baseline characteristics between diabetic and nondiabetic cohorts identified in 2000-2005

\begin{tabular}{|c|c|c|c|c|c|}
\hline & \multicolumn{2}{|c|}{ Comparison group } & \multicolumn{2}{|c|}{ Diabetes group } & \multirow[t]{3}{*}{$P$ value } \\
\hline & \multicolumn{2}{|c|}{$N=78,500$} & \multicolumn{2}{|c|}{$N=19,625$} & \\
\hline & $n$ & $\%$ & $n$ & $\%$ & \\
\hline Women & 34,760 & 44.28 & 8,690 & 44.28 & 1 \\
\hline \multicolumn{6}{|l|}{ Age, years } \\
\hline $20-39$ & 8,316 & 10.59 & 2,079 & 10.59 & 1 \\
\hline $40-64$ & 48,276 & 61.50 & 12,069 & 61.50 & \\
\hline$\geq 65$ & 21,908 & 27.91 & 5,477 & 27.91 & \\
\hline Mean $\pm \mathrm{SD}$ & \multicolumn{2}{|l|}{$56 \pm 13.7$} & \multicolumn{2}{|c|}{$56 \pm 13.4$} & \\
\hline \multicolumn{6}{|l|}{ Comorbidity ${ }^{\mathrm{b}}$} \\
\hline Helicobacter infection & 26 & 0.03 & 12 & 0.06 & 0.07 \\
\hline Non-acute gastritis & 3,764 & 4.79 & 1,124 & 5.73 & $<0.0001$ \\
\hline Pernicious anemia & 62 & 0.08 & 19 & 0.10 & 0.44 \\
\hline Gastric polyp & 142 & 0.18 & 29 & 0.15 & 0.32 \\
\hline Vagotomy & 124 & 0.16 & 17 & 0.09 & 0.02 \\
\hline Partial gastrectomy & 137 & 0.17 & 41 & 0.21 & 0.31 \\
\hline GERD & 282 & 0.36 & 101 & 0.51 & 0.002 \\
\hline Gastric ulcer & 6,346 & 8.08 & 1,892 & 9.64 & $<0.0001$ \\
\hline Obesity & 28 & 0.04 & 66 & 0.34 & $<0.0001$ \\
\hline Asbestosis & 54 & 0.07 & 13 & 0.07 & 0.90 \\
\hline Pneumoconiosis & 581 & 0.74 & 155 & 0.79 & 0.47 \\
\hline
\end{tabular}


Table 2 Hazard ratio for incidence of gastric cancer estimated by age, sex, and follow-up duration in the diabetic group versus the nondiabetic group

\begin{tabular}{|c|c|c|c|c|c|c|c|c|}
\hline & \multicolumn{3}{|l|}{ Non-diabetes } & \multicolumn{3}{|l|}{ Diabetes } & \multicolumn{2}{|c|}{ Hazard ratio $(95 \% \mathrm{CI})$} \\
\hline & No. of events & $\begin{array}{l}\text { Person- } \\
\text { years }\end{array}$ & $\begin{array}{l}\text { Incidence } \\
\text { rate }^{\mathrm{a}}\end{array}$ & No. of events & $\begin{array}{l}\text { Person- } \\
\text { years }\end{array}$ & $\begin{array}{l}\text { Incidence } \\
\text { rate }^{\mathrm{b}}\end{array}$ & Crude & Adjusted $^{\mathrm{b}}$ \\
\hline All & 216 & 444,545 & 4.86 & 47 & 10,8260 & 4.34 & $0.89(0.65-1.22)$ & $0.90(0.65-1.23)$ \\
\hline \multicolumn{9}{|l|}{ Sex } \\
\hline Women & 81 & 199,192 & 4.07 & 21 & 48,626 & 4.32 & $1.03(0.63-1.67)$ & $1.04(0.64-1.7)$ \\
\hline Men & 135 & 245,353 & 5.50 & 26 & 59,634 & 4.36 & $0.80(0.53-1.22)$ & $0.81(0.53-1.23)$ \\
\hline \multicolumn{9}{|c|}{ Age, years } \\
\hline $20-39$ & 1 & 47,857 & 0.21 & 1 & 11,928 & 0.84 & $4.12(0.26-65.8)$ & $4.23(0.26-67.6)$ \\
\hline $40-64$ & 70 & 280,281 & 2.50 & 22 & 68,588 & 3.21 & $1.22(0.75-1.99)$ & $1.20(0.74-1.96)$ \\
\hline$\geq 65$ & 145 & 116,406 & 12.5 & 24 & 27,744 & 8.65 & $0.71(0.46-1.10)$ & $0.71(0.46-1.10)$ \\
\hline \multicolumn{9}{|c|}{ Follow-up years } \\
\hline$<5$ & 162 & 352,608 & 4.59 & 25 & 86,519 & 2.89 & $0.63(0.41-0.96)$ & $0.63(0.42-0.97)$ \\
\hline$\geq 5$ & 54 & 91,937 & 5.87 & 22 & 21,742 & 10.1 & $1.71(1.03-2.83)$ & $1.76(1.06-2.91)$ \\
\hline
\end{tabular}

${ }^{a}$ Incidence rate per 10,000 person-years

b Hazard ratio (HR) adjusted for age, sex, gastric polyp, partial gastrectomy, gastric ulcer, pneumoconiosis

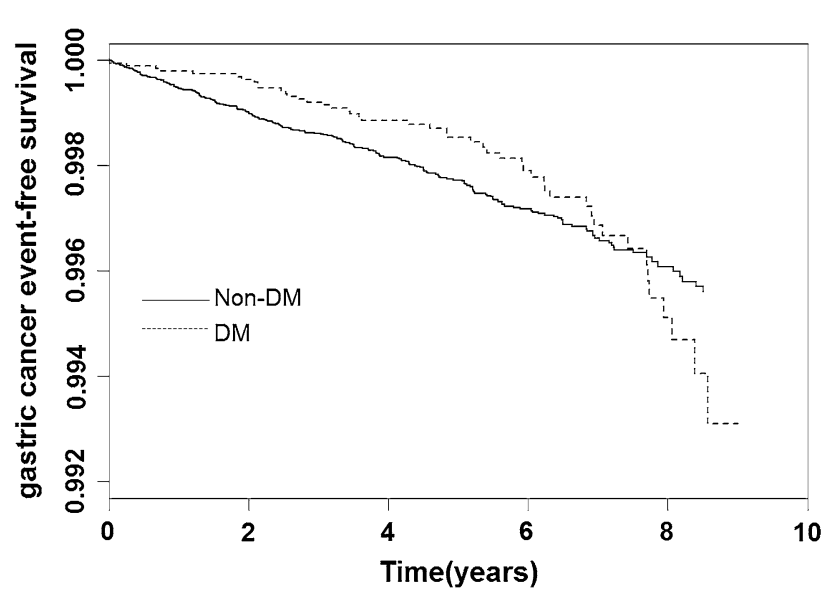

Fig. 1 Kaplan-Meier event-free survival for incidence of gastric cancer in patients with diabetes $(D M)$ and without diabetes (non-DM)

women and increased with age. Among various types of comorbidity, significantly elevated risks appeared in those with gastric polyp (adjusted $\mathrm{HR}=7.44,95 \% \mathrm{CI}=$ 2.73-20.30), partial gastrectomy (adjusted $\mathrm{HR}=6.25$, $95 \% \mathrm{CI}=2.56-15.25$ ), and pneumoconiosis (adjusted $\mathrm{HR}=2.30,95 \% \mathrm{CI}=1.08-4.89$ ).

Table 4 shows the adjusted HR of gastric cancer for diabetic patients prescribed with the antidiabetic drug compared with those not prescribed. The mean duration of prescription for each drug was in a range of 1-2 years, shortest for nateglinide [mean (median), 0.7 (0.3) years] and longest for metformin and sulfonylurea [mean (median) (both), 2.2 (1.8) years]. Patients prescribed for alpha-glucosidase inhibitor medication was associated with a $62 \%$ reduced risk of gastric cancer $(95 \% \mathrm{CI}=0.15-0.96)$.

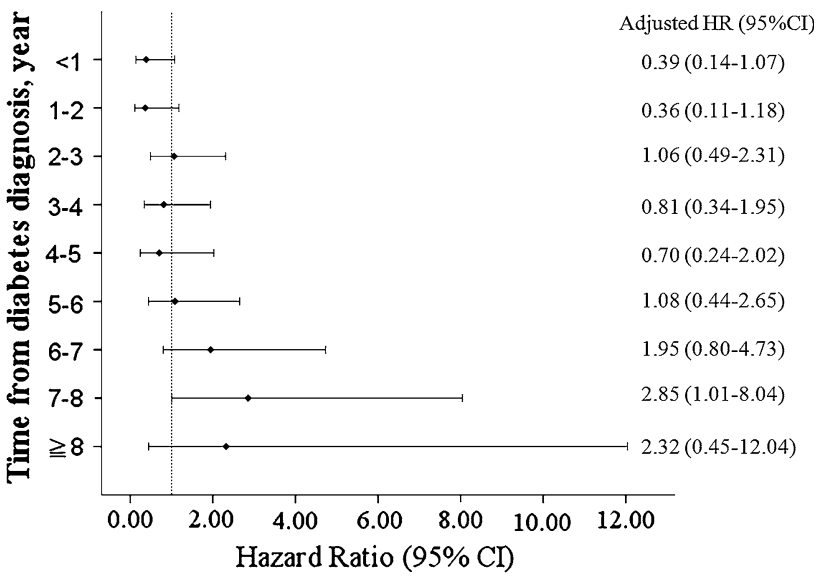

Fig. 2 Hazard ratios (95\% CIs) for incidence of gastric cancer in patients with diabetes versus subjects in the nondiabetic group, by duration of diabetic diagnosis. The models were adjusted for age, sex, gastric polyp, gastrectomy, gastric ulcer, and pneumoconiosis

The adjusted HRs for patients treated with insulin, metformin, sulfonylureas, thiazolidinediones, and repaglinide were approximately 0.80 . Patients treated with nateglinide had an adjusted HR of 1.93, but the HRs for all antidiabetic agents except alpha-glucosidase inhibitors were not statistically significant.

\section{Discussion}

In this study, we did not find a significant difference in gastric cancer incidence between diabetic and nondiabetic groups. However, our observations showed that the association 
Table 3 Characteristics of patients and comorbidity associated with gastric cancer

\begin{tabular}{lcc}
\hline Variable & \multicolumn{2}{l}{ Hazard ratio (95 \% CI) } \\
\cline { 2 - 3 } & Crude & Adjusted $^{\mathrm{a}}$ \\
\hline Sex & 1 (reference) & 1 (reference) \\
Women & $1.29(1.00-1.65)$ & $1.47(1.151 .89)$ \\
Men & & \\
Age, years & 1 (reference) & 1 (reference) \\
20-39 & $7.89(1.94-32.0)$ & $7.93(1.95-32.2)$ \\
40-64 & $35.4(8.77-142.6)$ & $35.02(8.68-141.4)$ \\
$\geq 65$ & & \\
Comorbidity (yes vs. no) & & $1.07(0.64-1.80)$ \\
Non-acute gastritis & $1.42(0.86-2.36)$ & $4.11(0.58-29.4)$ \\
Pernicious anemia & $6.06(0.86-43.0)$ & $7.44(2.73-20.3)$ \\
Gastric polyp & $10.7(3.99-28.8)$ & $6.25(2.56-15.3)$ \\
Partial gastrectomy & $11.6(4.80-28.2)$ & $1.16(0.16-8.32)$ \\
GERD & $1.55(0.22-11.0)$ & $1.13(0.77-1.66)$ \\
Gastric ulcer & $1.69(1.16-2.46)$ & $3.46(0.46-26.4)$ \\
Asbestosis & $5.20(0.73-37.0)$ & $2.30(1.08-4.89)$ \\
Pneumoconiosis & $3.72(1.76-7.88)$ &
\end{tabular}

The entries "reference" indicate the reference group

a Adjusted for age, sex, gastric polyp, partial gastrectomy, gastric ulcer, pneumoconiosis

b No patient experienced gastric cancer during the follow-up period among those with Helicobacter infection, vagotomy, or obesity

between diabetes and incidence of gastric cancer may vary over time. Relative to nondiabetic subjects, diabetic patients had a lower incidence of gastric cancer during earlier years but had greater risk past 5 years after diabetes diagnosis. However, this result was a hypothesis-generating observation, as the 5-year duration was not pre-specified. Further studies are needed to clarify the possible interaction between diabetes and survival time at 5 years.

Previous epidemiological studies showed conflicting findings in the relationship between gastric cancer risk and diabetes. The variation in results among studies may result from differences in study designs, including definition of diabetes and enrolled ethnic populations or regions. A Japanese prospective cohort study in a general population showed that a modest increased fasting plasma glucose is associated with increased risk of gastric cancer [16]. Another Japanese study identified diabetes using selfreported data and found a positive association between diabetes and subsequent risk of developing gastric cancer in women but not in men [7]. A large Korean study suggested that diabetes, identified by self-reported diabetes medication or a fasting blood glucose level of at least $126 \mathrm{mg} / \mathrm{dl}$, was associated with increased mortality from gastric cancer in men but not in women [8]. In contrast, a recent investigation in U.S. male veterans revealed a decreased risk of gastric cancer in black men with diabetes [12], but not in white men, indicating ethnical variations in the diabetes-gastric cancer association. Other studies conducted in Austria [18] and Denmark [11] revealed no association between diabetes and risk of gastric cancer.

To the best of our knowledge, limited data on the relationship between duration of diabetes and the occurrence of gastric cancer have been reported. Some explanations could be considered to interpret our observations on the changes in the diabetes-gastric cancer association over time. First, increasing diabetic duration elevates the risk of developing gastric cancer in diabetic subjects. A positive association between diabetes duration and cancer incidence has been observed in studies investigating hepatocellular carcinoma [19] and pancreas cancer [20]. Inoue et al. [7] found that the increased risk of gastric cancer associated with dibates was more pronounced after excluding cases diagnosed as having gastric cancer within 5 years since the baseline survey. A recent study in Taiwan revealed that diabetes duration was an independent predictor of mortality from gastric cancer only when subjects who died of gastric cancer within 5 years after diabetes diagnosis were excluded from the analysis [3]. It is reasonable to observe a positive relationship between diabetes duration and cancer development if diabetes exposure promotes tumorigenesis. Longer diabetes duration is usually linked to increasing risk of insulin resistance and hyperinsulinemia. Insulin has an effect on promoting cell growth and proliferation. Previous investigations suggested that insulin resistance and hyperinsulinemia may increase risk in some types of cancer [21]. Second, selection of antidiabetic agents may influence the risk of gastric cancer. In the early diabetic treatment period, Metformin monotherapy or combination therapy with sulfonylureas is usually the initial prescription in Taiwan. However, insulin therapy is usually required for subjects who have diabetes diagnosis for 5 years and longer because blood glucose control is inadequate with only oral medication. Several studies have supported that Metformin has anticancer effects [22], and $83 \%$ of our diabetic subjects were prescribed Metformin. However, evidence suggests that insulin therapy might increase cancer risk [4]. An increased proportion of insulin therapy might be the cause of increasing risk of gastric cancer at the later treatment period. Third, long-term treatment of anti-diabetes agents might be responsible for the observed rise in risk of gastric cancer at the later observation period, in the sense of a cumulating risk factor such as many exposures in epidemiology. Fourth, the symptoms of gastric cancer would be hidden and diagnosis might be late in diabetic patients with gastric cancer. Therefore, the numbers of gastric cancer patients increase after more than 5 years. Fifth, our analysis stratified by follow-up duration has to be interpreted cautiously, as the number of gastric cancer cases was small in the stratifications (fewer than ten events in each of the 1-year intervals). 
Table 4 Cox model measured hazard ratios and $95 \%$ confidence intervals of gastric cancer associated with anti-diabetic drugs among diabetic patients identified in 2000-2005

\begin{tabular}{|c|c|c|c|c|c|c|c|}
\hline & \multirow[t]{2}{*}{$n$} & \multirow{2}{*}{$\begin{array}{l}\text { Strength of drug exposure, } \\
\text { mean (median) } \\
\text { Years of use }\end{array}$} & \multirow{2}{*}{$\begin{array}{l}\text { Cases of gastric } \\
\text { cancer, number }\end{array}$} & \multirow[t]{2}{*}{ Person-years } & \multirow[t]{2}{*}{ Incidence $^{\mathrm{a}}$} & \multicolumn{2}{|c|}{ Hazard ratio $(95 \% \mathrm{CI})$} \\
\hline & & & & & & Crude & Adjusted \\
\hline \multicolumn{8}{|l|}{ Insulin } \\
\hline Non-use & 7,879 & - & 19 & 40,237 & 4.72 & 1 (reference) & 1 (reference) \\
\hline Use & 11,746 & $1.4(0.8)$ & 28 & 68,024 & 4.12 & $0.77(0.43-1.38)$ & $0.8(0.45-1.44)$ \\
\hline \multicolumn{8}{|l|}{ Metformin } \\
\hline Non-use & 3,201 & - & 9 & 15,071 & 5.97 & 1 (reference) & 1 (reference) \\
\hline Use & 16,424 & $2.2(1.8)$ & 38 & 93,190 & 4.08 & $0.62(0.30-1.29)$ & $0.82(0.39-1.71)$ \\
\hline \multicolumn{8}{|c|}{ Sulfonylurea } \\
\hline Non-use & 3,400 & - & 8 & 16,072 & 4.98 & 1 (reference) & 1 (reference) \\
\hline Use & 16,225 & $2.2(1.8)$ & 39 & 92,188 & 4.23 & $0.74(0.35-1.59)$ & $0.83(0.38-1.79)$ \\
\hline \multicolumn{8}{|c|}{ Thiazolidinedione } \\
\hline Non-use & 15,780 & - & 39 & 84,527 & 4.61 & 1 (reference) & 1 (reference) \\
\hline Use & 3,845 & $1.2(0.8)$ & 8 & 23,734 & 3.37 & $0.66(0.31-1.42)$ & $0.82(0.38-1.77)$ \\
\hline \multicolumn{8}{|c|}{ Alpha-glucosidase inhibitor } \\
\hline Non-use & 15,153 & - & 42 & 81,377 & 5.16 & 1 (reference) & 1 (reference) \\
\hline Use & 4,472 & $1.1(0.6)$ & 5 & 26,883 & 1.86 & $0.34(0.13-0.85)$ & $0.38(0.15-0.96)$ \\
\hline \multicolumn{8}{|l|}{ Nateglinide } \\
\hline Non-use & 18,805 & - & 43 & 103,540 & 4.15 & 1 (reference) & 1 (reference) \\
\hline Use & 820 & $0.7(0.3)$ & 4 & 4,720 & 8.47 & $2.02(0.72,5.62)$ & $1.93(0.69-5.39)$ \\
\hline \multicolumn{8}{|c|}{ Repaglinide } \\
\hline Non-use & 16,832 & - & 41 & 91,584 & 4.48 & 1 (reference) & 1 (reference) \\
\hline Use & 2,793 & $1.0(0.5)$ & 6 & 16,676 & 3.60 & $0.76(0.32,1.79)$ & $0.72(0.31-1.70)$ \\
\hline
\end{tabular}

HR adjusted for age and sex

The entries "reference" indicate the reference group

a Incidence per 10,000 person-years

The mechanism of developing gastric cancer in diabetic patients is unclear. In addition to the effect on carcinogenesis caused by insulin resistance, the role of insulin-like growth factors (IGF) receptors on cancer cells has also drawn attention in recent decades. The IGF-1 signaling pathway may exert effects on cellular proliferation and apoptosis [23]. High circulating IGF-I levels were associated with an increased risk of prostate, breast, and colon cancer [24]. Uncontrolled hyperglycemia might encourage tumor growth, but there is not adequate evidence to support this hypothesis, which needs further exploration [5].

Our observation indicated that among diabetic patients, users of alpha-glucosidase inhibitor had $62 \%$ of decreased risk of gastric cancer relative to nonusers. Decrease in risk was also noted in users of insulin, metformin, sulfonylureas, thiazolidinediones, and repaglinide, but the association was not statistically significant, possibly because statistical power was insufficient to detect the moderate association. To our best knowledge there has been no study concerning the protective effect of alpha-glucosidase inhibitors in reducing the risk of gastric cancer. A matched case-control study has reported no significant association between pharmacological treatment with acarbose and cancer incidence, but no data on gastric cancer were provided [25]. Alpha-glucosidase inhibitors are known to interrupt starch digestion, and starchy foods are a suggested risk factor for gastric cancer in some studies [26]. Whether lower gastric cancer risk in alpha-glucosidase inhibitor users could be explained by inhibited starchy food digestion remains in doubt; it was not thought that carcinogenesis could be caused in such a short period of time. Some studies have suggested that patients receiving sulfonylureas and insulin are at an increased risk of developing cancers [4]. On the other hand, a protective effect of metformin against cancer growth has been reported in both experimental and population-based studies [27-29]. Evidence concerning the role of thiazolidinediones on cancer risk was sparse and controversial; some reports suggested a preventive effect but some did not [4].

This study has the strength of using a large cohort selected from a nationwide representative database, which may reduce the potential of selection bias. The 
comprehensive pharmacological data in NHIRD allow us to investigate the association between anti-diabetics and gastric cancer. However, several limitations of this study should also be considered. First, claims databases do not contain information on an individual's biochemical and anthropometric data. Thus, we were unable to assess levels of blood glucose, HbA1C, and body mass index. Second, underdiagnosis of diseases may occur when screening tests are not performed, particularly for asymptomatic medical conditions. Some conventional risk factors of gastric cancer, such as Helicobacter infection and obesity, were less prevalent in this study than in other studies undertaken in Taiwan $[30,31]$. We speculated that the low prevalence of Helicobacter pylori is attributable to a low screening rate. In addition, we believed that most subjects with obesity have not been registered during clinical practice in Taiwan. Physicians might register an obesity diagnosis only when severe obesity is noted and aggressive intervention is necessary. The underestimation of these risk factors could lead to a certain degree of overestimating the diabetesgastric cancer association. Third, a median follow-up of 5.5 years may not account for the much longer induction period of gastric cancer. However, the increased risk of gastric cancer in subjects with with diabetes duration of 5 years or longer suggests more prominent gastric cancer risks may be expected in diabetic cases with increasing years of follow up. Fourth, in the analyses on the effect of anti-diabetic drugs, we were unable to assess the interaction between multiple drugs because of the small number of cancer cases. Future studies with a large number of subjects are needed to clarify the issue.

This population-based study in Taiwan suggested that the association between diabetes and subsequent risk of gastric cancer may vary over time. The increased risk of gastric cancer was observed in patients with longer duration of diabetes but not in earlier years of diabetes diagnosis. Further studies with larger number of study subjects and events are required to validate our findings and to investigate the timing of increased risk. Additional investigations designed to assess the protective effect of anti-diabetic medications on gastric cancer are also warranted.

\footnotetext{
Acknowledgments This study was supported in part by the Executive Yuan National Science Council (Grant number NSC 99-2621M-039 -001), Clinical Trial and Research Center of Excellence of the Department of Health (Grant number DOH100-TD-B-111-004), Taiwan Department of Health Cancer Research Center of Excellence (Grant number DOH100-TD-C-111-005), and the China Medical University Hospital (Grant number 1MS1). The funding agencies had no role in the study design, data collection and analysis, decision to publish, or preparation of the manuscript.
}

Conflict of interest The authors have no conflict of interest.

\section{References}

1. Crew KD, Neugut AI. Epidemiology of gastric cancer. J Gastroenterol. 2006;12:354-62.

2. Amiri M, Janssen F, Kunst AE. The decline in stomach cancer mortality: exploration of future trends in seven European countries. Eur J Epidemiol. 2011;26:23-8.

3. Tseng C-H. Diabetes conveys a higher risk of gastric cancer mortality despite an age-standardised decreasing trend in the general population in Taiwan. Gut. 2011;60:774-9.

4. Chowdhury TA. Diabetes and cancer. Q J Med. 2010;103: 905-15.

5. Giovannucci E, Harlan DM, Archer MC, Bergenstal RM, Gapstur SM, Habel LA, et al. Diabetes and cancer: a consensus report. CA Cancer J Clin. 2010;60:207-21.

6. Barone BB, Yeh H-C, Snyder CF, Peairs KS, Stein KB, Derr RL, et al. Long-term all-cause mortality in cancer patients with preexisting diabetes mellitus. JAMA. 2008;300:2754-64.

7. Inoue M, Iwasaki M, Otani T, Sasazuki S, Noda M, Tsugane S. Diabetes mellitus and the risk of cancer: results from a large-scale population-based cohort study in Japan. Arch Intern Med. 2006;166:1871.

8. Jee SH, Ohrr H, Sull JW, Yun JE, Ji M, Samet JM. Fasting serum glucose level and cancer risk in Korean men and women. JAMA. 2005;293:194-202.

9. Rousseau M-C, Parent M-É, Pollak MN, Siemiatycki J. Diabetes mellitus and cancer risk in a population-based case-control study among men from Montreal, Canada. Int J Cancer. 2006;118:2105-9.

10. Hemminki K, Li X, Sundquist J, Sundquist K. Risk of cancer following hospitalization for type 2 diabetes. Oncologist. 2010; 15:548-55.

11. Wideroff L, Gridley G, Chow W-H, Linet M, Mellemkjaer L, Olsen JH, et al. Cancer incidence in a population-based cohort of patients hospitalized with diabetes mellitus in Denmark. J Natl Cancer Inst. 1997;89:1360-5.

12. Atchison EA, Gridley G, Carreon JD, Leitzmann MF, McGlynn KA. Risk of cancer in a large cohort of U.S. veterans with diabetes. Int J Cancer. 2011;128:635-43.

13. Coughlin SS, Calle EE, Teras LR, Petrelli J, Thun MJ. Diabetes mellitus as a predictor of cancer mortality in a large cohort of US adults. Am J Epidemiol. 2004;159:1160-7.

14. Zhou X, Qiao Q, Zethelius B, Pyörälä K, Söderberg S, Pajak A, et al. Diabetes, prediabetes and cancer mortality. Diabetologia. 2010;53:1867-76.

15. Stattin P, Björ O, Ferrari P, Lukanova A, Lenner P, Lindahl B, et al. Prospective study of hyperglycemia and cancer risk. Diabetes Care. 2007;30:561-7.

16. Yamagata H, Kiyohara Y, Nakamura S, Kubo M, Tanizaki Y, Matsumoto T, et al. Impact of fasting plasma glucose levels on gastric cancer incidence in a general Japanese population. Diabetes Care. 2005;28:789-94.

17. Lu JFR, Hsiao WC. Does universal health insurance make health care unaffordable? Lessons from Taiwan. Health Aff. 2003; 22:77.

18. Rapp K, Schroeder J, Klenk J, Ulmer H, Concin H, Diem G, et al. Fasting blood glucose and cancer risk in a cohort of more than 140,000 adults in Austria. Diabetologia. 2006;49:945-52.

19. Hassan MM, Curley SA, Li D, Kaseb A, Davila M, Abdalla EK, et al. Association of diabetes duration and diabetes treatment with the risk of hepatocellular carcinoma. Cancer (Phila). 2010;116: 1938-46.

20. Everhart J, Wright D. Diabetes mellitus as a risk factor for pancreatic cancer. JAMA. 1995;273:1605-9.

21. Pisani P. Hyper-insulinaemia and cancer, meta-analyses of epidemiological studies. Arch Physiol Biochem. 2008;114:63-70. 
22. Libby G, Donnelly LA, Donnan PT, Alessi DR, Morris AD, Evans JMM. New users of metformin are at low risk of incident cancer. Diabetes Care. 2009;32:1620.

23. Renehan AG, Zwahlen M, Minder C, O'Dwyer ST, Shalet SM, Egger M. Insulin-like growth factor (IGF)-I, IGF binding protein3 , and cancer risk: systematic review and meta-regression analysis. Lancet. 2004;363:1346-53.

24. Pollak MN, Schernhammer ES, Hankinson SE. Insulin-like growth factors and neoplasia. Nat Rev Cancer. 2004;4:505-18.

25. Monami M, Lamanna C, Balzi D, Marchionni N, Mannucci E. Sulphonylureas and cancer: a case-control study. Acta Diabetol. 2009;46:279-84.

26. De Stefani E, Correa P, Boffetta P, Deneo-Pellegrini H, Ronco AL, Mendilaharsu M. Dietary patterns and risk of gastric cancer: a factor analysis in Uruguay. Gastric Cancer. 2004;7: 211-20.
27. Cantrell LA, Zhou C, Mendivil A, Malloy KM, Gehrig PA, BaeJump VL. Metformin is a potent inhibitor of endometrial cancer cell proliferation: implications for a novel treatment strategy. Gynecol Oncol. 2010;116:92-8.

28. Currie C, Poole C, Gale E. The influence of glucose-lowering therapies on cancer risk in type 2 diabetes. Diabetologia. 2009;52:1766-77.

29. Bodmer M, Meier C, Krähenbühl S, Jick SS, Meier CR. Longterm metformin use is associated with decreased risk of breast cancer. Diabetes Care. 2010;33:1304-8.

30. Lin JT, Wang LY, Wang JT, Wang TH, Yang CS, Chen CJ. A nested case-control study on the association between Helicobacter pylori infection and gastric cancer risk in a cohort of 9775 men in Taiwan. Anticancer Res. 1995;15:603-6.

31. Chu NF. Prevalence of obesity in Taiwan. Obes Rev. 2005; $6: 271-4$. 